\title{
ADIÇÃO DE Zn E ABSORÇÃO, TRANSLOCAÇÃO E UTILIZAÇÃO DE Zn E P POR CULTIVARES DE CAFEEIRO
}

\author{
Roberto dos Anjos Reis Jr. ${ }^{1 *}$; Hermínia E. P. Martinez² \\ ${ }^{1}$ Fundação Chapadão, C.P. 39 - CEP: 79560-000 - Chapadão do Sul, MS. \\ ${ }^{2}$ Depto. de Fitotecnia, UFV - CEP: 365700-000 - Viçosa, MG. \\ *Autor correspondente <reisjr@hotmail.com>
}

\begin{abstract}
RESUMO: A crescente exigência de nutrientes, especialmente micronutrientes, pelos cultivares de café vem exigindo uma melhor compreensão da dinâmica desses nutrientes no sistema solo-planta. Com o objetivo de avaliar o efeito de concentrações de $\mathrm{Zn}\left(0,4 ; 1,3\right.$ e 2,3 $\left.\mu \mathrm{mol} \mathrm{Zn} \mathrm{L}{ }^{-1}\right)$ na solução nutritiva sobre o crescimento, produção de matéria seca, eficiência de absorção, translocação e utilização de Zn e $P$ em dois cultivares de café (Coffea arabica cv. Catuaí e Coffea canephora cv. Conilon), foi realizado um experimento sob delineamento inteiramente casualizado com esquema fatorial $3 \times 2$ (três doses de $\mathrm{Zn}$ e dois cultivares) com três repetições. Plantas de café dos dois cultivares estudados foram cultivadas em casa de vegetação, com solução nutritiva completa por 50 dias, após os quais receberam solução nutritiva modificada com três doses de Zn. Aos 114 dias após a transferência para a solução nutritiva modificada, as plantas foram coletadas e separadas em folhas superiores, inferiores, caule e raízes. Foram avaliadas altura do caule, número de ramificações laterais, área foliar, produção de matéria seca e conteúdos de $\mathrm{P} e$ $\mathrm{Zn}$ nas diversas partes da planta, além das eficiências de absorção, translocação e utilização de $\mathrm{P}$ e Zn. A dose 2,3 $\mu \mathrm{mol} \mathrm{Zn} \mathrm{L}^{-1}$ proporcionou os maiores valores para as características de crescimento avaliadas, tanto para o cultivar Catuaí, quanto para Conilon. Os maiores conteúdos de Zn observados no Catuaí indicam que este é mais exigente em $\mathrm{Zn}$ que o Conilon na fase de mudas. O Conilon apresentou menores eficiências de absorção e translocação e maior eficiência de utilização de Zn, refletindo sua menor demanda por este nutriente. As concentrações de $\mathrm{Zn}$ empregadas neste experimento não promoveram interação Zn-P.
\end{abstract}

Palavras-chave: C. arabica cv. Catuaí, C. canephora cv. Conilon, café

\section{Zn ADDITION AND Zn AND P UPTAKE, TRANSLOCATION AND UTILIZATION BY COFFEE CULTIVARS}

\begin{abstract}
The increasing nutrient requirement of coffee varieties, specially of micronutrients, is claiming for a better understanding of the dynamics of these nutrients in the soil-plant system. Having this in mind a greenhouse experiment in a completely randomized design, $3 \times 2$ factorial scheme and three replications was conducted to evaluate the effect of three $\mathrm{Zn}$ levels $\left(0.4,1.3\right.$ and $\left.2.3 \mu \mathrm{mol} \mathrm{L}^{-1}\right)$ and two coffee species (Coffea arabica cv. Catuaí and Coffea canephora cv. Conilon) on: (i) growth characteristics; (ii) Zn and P contents; and (iii) Zn and P uptake, translocation and utilization efficiencies. Plants were grown in complete nutrient solution along 50 days, when they were transferred to three $\mathrm{Zn}$ levels. Plants were harvested and separated in leaves, stem and roots 114 days after the transference. Stem height, leaf area, dry matter yield, $\mathrm{Zn}$ and $\mathrm{P}$ contents, and $\mathrm{Zn}$ and $\mathrm{P}$ uptake, translocation and utilization efficiencies were evaluated. The largest growth characteristics were observed with $2.3 \mu \mathrm{mol} \mathrm{L}^{-1}$. The highest Coffea arabica cv Catuaí $\mathrm{Zn}$ contents suggest that this species has higher $\mathrm{Zn}$ demand than Coffea canephora cv. Conilon. Conilon cultivar showed the lowest $\mathrm{Zn}$ uptake and translocation efficiencies and the highest $\mathrm{Zn}$ utilization efficiency; what reflects its lower $\mathrm{Zn}$ demand. The $\mathrm{Zn}-\mathrm{P}$ interaction was not observed with the $\mathrm{Zn}$ levels employed in this experiment.
\end{abstract}

Key words: C. arabica cv. Catuaí, C. canephora cv. Conilon, coffee

\section{INTRODUÇÃO}

A crescente demanda de nutrientes pelos cultivares de café, cada vez mais produtivos, assim como a expansão de lavouras de café para solos de baixa fertilidade, vêm exigindo uma melhor compreensão da dinâmica dos nutrientes na cultura do café, para que sejam evitados problemas de deficiência nutricional, que muito prejudicam a eficiência dos programas de adubação.
A cultura do café é exigente em micronutrientes, especialmente em relação ao zinco. Este micronutriente tem comprometido a produtividade da cafeicultura brasileira pois nossos solos são, de modo geral, pobres neste elemento. Isto tem sido demonstrado nos levantamentos do estado nutricional do cafeeiro realizados por Lott et al. (1961), Gallo et al. (1967), Gallo et al. (1970) e Garcia et al. (1983), que constataram que a maioria das lavouras avaliadas apresentavam deficiência de zinco. 
O zinco possui nítida importância na nutrição mineral do café, principalmente em relação à sua qualidade (IBC, 1985). Sob deficiência de zinco, os internódios do cafeeiro vão encurtando da base do ramo para a ponta (Malavolta et al., 1993) e as folhas novas tornam-se menores, levemente coriáceas e quebradiças (IBC, 1985). A deficiência de zinco é uma das mais generalizadas e limitantes para a cultura do café, causando a morte dos ponteiros, superbrotamento (Malavolta et al., 1993) e limitação do crescimento da parte aérea (IBC, 1985). Graves distúrbios tais como menor pegamento de florada e frutos menores são resultados da deficiência de zinco na cultura do café (Malavolta et al., 1993).

Problemas na nutrição com zinco têm sido observados em decorrência de crescentes adubações fosfatadas, fazendo com que a interação ZnxP seja objeto de vários estudos (Bingham, 1963; Igue \& Bornesmiza, 1967; Olsen, 1972; Pasricha et al., 1987; Souza \& Ferreira, 1991). Altas doses de fósforo parecem diminuir a concentração de zinco na parte aérea, além do que aplicações de zinco afetam a concentração de fósforo nos tecidos foliares (Marques, 1990). Os mecanismos que regulam a interação entre $\mathrm{Zn}$ e $\mathrm{P}$ ainda são controvertidos. Bingham (1963) concluiu que a interação entre zinco e fósforo era um problema de solo e não estava ligado à fisiologia das plantas. Entretanto, Igue \& Bornemisza (1967) concluíram que o antagonismo ocorria na raiz. Olsen (1972), por sua vez, destacou como possíveis mecanismos para explicar as relações antagônicas: interação PxZn no solo; redução da taxa de translocação de $Z n$ das raízes para a parte aérea; simples efeito de diluição sobre a concentração de zinco na parte aérea devido ao crescimento em resposta ao fósforo; e desordens metabólicas nas células vegetais, relacionadas com o desequilíbrio entre zinco e fósforo interferindo na função metabólica do zinco em certos sítios celulares.

As modificações na morfologia e eficiência nutricional da planta diante da interação entre zinco e fósforo podem contribuir para o aumento da capacidade de produção de matéria seca com os nutrientes disponíveis e, conseqüentemente, aumentar a produção. Assim, a avaliação de cultivares de café com maior eficiência de absorção, translocação e utilização de zinco passa a ser importante para evitar que situações de deficiência deste micronutriente possa ocorrer.

O objetivo do presente trabalho foi avaliar o efeito de concentrações de Zn sobre o crescimento, produção de matéria seca e as eficiências de absorção, translocação e utilização de Zn e P em Coffea arabica L. cv. Catuaí e Coffea canephora cv. Conilon cultivadas em solução nutritiva.

\section{MATERIAL E MÉTODOS}

Plantas de Coffea arabica cv. Catuaí e Coffea canephora cv. Conilon, germinadas em papel toalha, foram cultivadas em solução nutritiva de Clark completa por 50 dias em casa-de-vegetação, após os quais receberam solução de Clark modificada e com doses de 0,$4 ; 1,3$ e $2,3 \mu \mathrm{mol} \mathrm{Zn} \mathrm{L}^{-1}$ por 114 dias.

As parcelas experimentais foram compostas por vasos de oito litros de capacidade, com duas plantas por vaso. Realizaram-se trocas periódicas das soluções a intervalos de 21,14 e 7 dias. O experimento constituiuse de um fatorial $3 \times 2$ (três doses de $Z n$ e dois cultivares de café), sob delineamento inteiramente casualizado com três repetições.

Ao final do experimento, as plantas de cada parcela foram coletadas e separadas em folhas inferiores e superiores, caule e raízes. Após avaliar a altura do caule $(A C)$, o número de ramificações laterais (NRL), o número de folhas (NF) e a área foliar (AF), o material (folhas inferiores e superiores, caule e raízes) foi submetido à secagem em estufa com circulação forçada de ar a $70^{\circ} \mathrm{C}$ por 72 horas, para que em seguida fosse avaliada a produção de matéria seca de folhas (MSF), de caules (MSC), de raízes (MSR), da parte aérea (MSPA) e da planta toda (MSP). Os valores de AC, NRL, NF, AF, MSF, MSC, MSR, MSPA e MSP foram expressos em relação a uma planta. A matéria seca de folhas superiores, folhas inferiores, caules e raízes foi submetida à digestão nitro-perclórica, seguida da determinação dos teores e conteúdos de $\mathrm{Zn}$ e $\mathrm{P}$ nas diferentes partes da planta. Calcularam-se as eficiências de absorção (conteúdo do nutriente na planta/produção de MSR), translocação (conteúdo do nutriente na parte aérea/conteúdo de nutriente na planta) e utilização (produção de $\mathrm{MSP} /$ conteúdo do nutriente na planta). O conteúdo de nutriente e a produção de matéria seca foram usados para calcular as eficiências descritas acima.

Os dados foram submetidos à análise de variância. O desdobramento do efeito de dose de $\mathrm{Zn}$ dentro de espécie somente foi realizado nos casos em que foram observadas interações significativa entre as variáveis independentes. As médias das variáveis dependentes foram comparadas por meio de teste de médias (Duncan a $5 \%$ ) quando foram observados efeitos de doses de Zn.

\section{RESULTADOS E DISCUSSÃO}

\section{Crescimento e produção de matéria seca}

Os cultivares apresentaram crescimento e produção de matéria seca diferenciados, evidenciados pela produção de matéria seca de raízes (MSR), altura de caule ( $A C)$, número de folhas (NF) e número de ramificações laterais (NRL). Isto é explicado pela característica de cada material, sendo o conilon de porte alto e sistema radicular robusto e o Catuaí de porte baixo mas com maior ramificação.

A produção de matéria seca de folhas e de caules não foi diferente entre o Catuaí e o Conilon, 
apresentando os valores médios de $15,5 \mathrm{~g}$ e $4,0 \mathrm{~g}$, respectivamente (Tabela 1). Entretanto, houve diferença de produção de MSR entre os cultivares $(P<0,05)$, pois o Conilon apresentou produção de $\operatorname{MSR}(6,2 \mathrm{~g})$ maior que o Catuaí $(4,4 \mathrm{~g})$. Mesmo com esta diferença de produção de MSR, não foram constatadas diferenças de produção de matéria seca de toda planta entre os cultivares, que apresentou valor médio de $23,6 \mathrm{~g}$.

O Conilon apresentou maior AC $(32,6 \mathrm{~cm})$ e menores NF $(36,1)$ e NRL $(0,9)$ que o Catuaí $(28,1 \mathrm{~cm}$; $58,5$ e 3,1 respectivamente) $(P<0,01)$ (Tabela 2$)$. A característica do Conilon de possuir folhas maiores que os cultivares arábicos (IBC, 1985) foi evidenciada neste trabalho, pois como a área foliar não foi diferente entre os cultivares, apresentando valor médio de $3468,6 \mathrm{~mm}^{2}$, o menor NF apresentado pelo Conilon foi compensado pela maior área de cada folha neste cultivar.

As doses de $\mathrm{Zn}$ influenciaram a produção de matéria seca de planta (MSP) $(P<0,05)$, que apresentou o maior valor $(26,6 \mathrm{~g})$ na dose de $2,3 \mu \mathrm{mol} \mathrm{L} \mathrm{L}^{-1}$ na solução nutritiva. $\mathrm{O}$ aumento da produção de MSP em função do fornecimento de $\mathrm{Zn}$ foi decorrente do aumento da produção de matéria seca de caule $(P<0,05)$, pois as produções de matéria seca de folhas e de raízes e não foram influenciadas pelas doses de $\mathrm{Zn}$, apresentando os valore médios de $15,5 \mathrm{~g}$ e $5,3 \mathrm{~g}$ respectivamente (Tabela 1). As concentrações de $Z n$ das soluções nutritivas não influenciaram $\mathrm{AC}, \mathrm{AF}$ e NF, que apresentaram os valores médios de $30,4 \mathrm{~cm}, 3468,6 \mathrm{~mm}^{2}$ e 47,3 folhas, respectivamente. Entretanto, NRL aumentou com as doses de $\operatorname{Zn}(P<0,05)$, alcançando a média de 2,5 ramificações por planta na dose de $2,3 \mu \mathrm{mol} \mathrm{L}^{-1}$ na solução nutritiva.

\section{Teores e conteúdo de Zinco}

Com exceção do conteúdo de zinco nas raízes (CZNR), que apresentou valor médio de $57,5 \mu \mathrm{g}$ por planta, os teores e conteúdos de $\mathrm{Zn}$ em todas as partes da planta foram maiores no Catuaí $(P<0,05)$, que apresentou teores de $\mathrm{Zn}$ nas folhas inferiores $(\mathrm{ZnFI})$, folhas superiores $(\mathrm{ZnFS})$, caules $(\mathrm{ZnC})$ e raízes $(\mathrm{ZnR})$ iguais a $6,6 \mathrm{mg} \mathrm{kg}^{-1} ; 8,0 \mathrm{mg} \mathrm{kg}^{-1} ; 16,6 \mathrm{mg} \mathrm{kg}^{-1}$ e $13,1 \mathrm{mg}$ $\mathrm{kg}^{-1}$ respectivamente e conteúdos de $\mathrm{Zn}$ nas folhas $(\mathrm{CZnF})$, caules $(\mathrm{CZnC})$ e parte aérea $(\mathrm{CZnPA})$ iguais a $110,0 \mu \mathrm{g} ; 62,3 \mu \mathrm{g}$ e $172 \mu \mathrm{g}$ respectivamente (Tabela 1). Os teores e conteúdos de $\mathrm{Zn}$ na matéria seca das plantas evidenciam maior exigência de $\mathrm{Zn}$ por parte do Catuaí.

Tabela 1 - Produção de matéria seca de folhas (MSF), caule (MSC), parte aérea (MSPA), raiz (MSR) e de toda a planta $(\mathrm{MSP})$, teores de zinco nas folhas inferiores $(\mathrm{ZnFI})$, folhas superiores $(\mathrm{ZnFS})$, caule $(\mathrm{ZnC})$ e raiz $(\mathrm{ZnR})$, conteúdos de zinco nas folhas inferiores $(\mathrm{CZnFI})$, folhas superiores $(\mathrm{CZnFS})$, folhas $(\mathrm{CZnF})$, caule $(\mathrm{CZnC})$, parte aérea $(C Z n P A)$ e raiz (CZnR), e eficiências de absorção (EFAZn), translocação (EFTZn) e utilização (EFUZn) de zinco de plantas de café dos cultivares Catuaí e Conilon cultivadas em solução nutritiva com diferentes concentrações de zinco.

\begin{tabular}{|c|c|c|c|c|c|c|c|c|c|c|c|c|c|c|c|}
\hline \multirow[t]{2}{*}{$\mathrm{Zn}$} & \multicolumn{3}{|c|}{ MSF } & \multicolumn{3}{|c|}{ MSC } & \multicolumn{3}{|c|}{ MSPA } & \multicolumn{3}{|c|}{ MSR } & \multicolumn{3}{|c|}{ MSP } \\
\hline & Catuaí & Conilon & Média & Catuaí & Conilon & Média & Catuaí & Conilon & Média & Catuaí & Conilon & Média & Catuaí & Conilon & Média \\
\hline$\mu \mathrm{mol} \mathrm{L}^{-1}$ & \multicolumn{15}{|c|}{ - } \\
\hline 0,4 & 14,4 & 14,6 & 14,5 & 3,4 & 3,0 & $3,2 \mathrm{~b}$ & 7,8 & 17,6 & $17,7 \mathrm{~b}$ & 4,6 & 5,2 & 4,9 & 21,2 & 20,5 & $20,9 b$ \\
\hline 1,3 & 15,3 & 15,2 & 15,3 & 4,0 & 4,0 & $4,0 a b$ & 19,3 & 19,3 & $19,3 a b$ & 4,3 & 5,9 & 5,1 & 23,2 & 23,3 & $23,3 a b$ \\
\hline 2,3 & 15,4 & 18,0 & 16,7 & 3,8 & 6,0 & $4,9 \mathrm{a}$ & 19,3 & 24,1 & $21,7 \mathrm{a}$ & 4,4 & 7,6 & 6,0 & 23,1 & 30,1 & $26,6 \mathrm{a}$ \\
\hline Média & 15,0 & 15,9 & 15,5 & 3,7 & 4,3 & 4,0 & 18,8 & 20,3 & 19,5 & $4,4 \mathrm{~B}$ & $6,2 \mathrm{~A}$ & 5,3 & 22,5 & 24,6 & 23,6 \\
\hline $\mathrm{Zn}$ & \multicolumn{3}{|c|}{$\mathrm{ZnFI}$} & \multicolumn{3}{|c|}{ ZnFS } & \multicolumn{3}{|c|}{$\mathrm{ZnC}$} & \multicolumn{3}{|c|}{$\mathrm{ZnR}$} & \multicolumn{3}{|c|}{ CZnFI } \\
\hline & Catuaí & Conilon & Média & Catuaí & Conilon & Média & Catuaí & Conilon & Média & Catuaí & Conilon & Média & Catuaí & Conilon & Média \\
\hline$\mu \mathrm{mol} \mathrm{L}^{-1}$ & \multicolumn{15}{|c|}{ - - } \\
\hline 0,4 & $3,7 \mathrm{~b}$ & 3,7 & 3,7 & 7,0 & 5,2 & 6,1 & $11,8 \mathrm{~b}$ & 9,2 & 10,5 & $5,3 \mathrm{c}$ & $4,7 \mathrm{~b}$ & 5,0 & $31,5 \mathrm{~b}$ & $29,3 b$ & 30,4 \\
\hline 1,3 & $11,4 \mathrm{a}$ & 3,7 & 7,6 & 8,4 & 5,7 & 7,0 & $14,0 \mathrm{~b}$ & 9,6 & 11,8 & $11,0 \mathrm{~b}$ & $8,5 b$ & 9,8 & $101,2 \mathrm{a}$ & $31,8 \mathrm{~b}$ & 66,5 \\
\hline 2,3 & $4,8 \mathrm{~b}$ & 6,1 & 5,5 & 8,7 & 6,3 & 7,5 & $24,0 \mathrm{a}$ & 11,2 & 17,6 & $23,1 \mathrm{a}$ & $14,1 \mathrm{a}$ & 18,6 & $39,5 \mathrm{~b}$ & $64,1 \mathrm{a}$ & 51,8 \\
\hline Média & $6,6 \mathrm{~A}$ & $4,5 \mathrm{~B}$ & 5,6 & $8,0 \mathrm{~A}$ & $5,7 \mathrm{~B}$ & 6,8 & $16,6 \mathrm{~A}$ & $10,0 \mathrm{~B}$ & 13,3 & $13,1 \mathrm{~A}$ & $9,1 \mathrm{~B}$ & 11,1 & $57,4 \mathrm{~A}$ & $41,8 \mathrm{~B}$ & 49,6 \\
\hline \multirow{2}{*}{$\mathrm{Zn}$} & \multicolumn{3}{|c|}{ CZnFS } & \multicolumn{3}{|c|}{$\mathrm{CZnF}$} & \multicolumn{3}{|c|}{$\mathrm{CZnC}$} & \multicolumn{3}{|c|}{$\mathrm{CZnPA}$} & \multicolumn{3}{|c|}{$\mathrm{CZnR}$} \\
\hline & Catuaí & Conilon & Média & Catuaí & Conilon & Média & Catuaí & Conilon & Média & Catuaí & Conilon & Média & Catuaí & Conilon & Média \\
\hline$\mu \mathrm{mol} \mathrm{L}^{-1}$ & \multicolumn{15}{|c|}{ - } \\
\hline 0,4 & 40,6 & 34,8 & $37,7 \mathrm{~b}$ & $72,1 \mathrm{c}$ & $64,1 \mathrm{~b}$ & 68,1 & 39,8 & 27,0 & $33,4 \mathrm{~b}$ & $111,9 \mathrm{~b}$ & $91,0 \mathrm{~b}$ & 101,5 & 23,7 & 24,5 & $24,1 \mathrm{c}$ \\
\hline 1,3 & 54,0 & 37,8 & $45,9 a b$ & $155,2 \mathrm{a}$ & $69,4 \mathrm{~b}$ & 112,4 & 55,3 & 38,1 & $46,7 \mathrm{~b}$ & $210,6 \mathrm{a}$ & $107,8 \mathrm{~b}$ & 159,2 & 47,5 & 48,6 & $48,1 \mathrm{~b}$ \\
\hline 2,3 & 63,0 & 46,6 & 54,8 a & $105,5 b$ & $110,7 a$ & 106,6 & 91,6 & 67,1 & 79,4 a & $194,1 \mathrm{a}$ & $177,9 \mathrm{a}$ & 186,0 & 101,9 & 98,5 & $100,2 \mathrm{a}$ \\
\hline Média & $52,6 \mathrm{~A}$ & $39,7 \mathrm{~B}$ & 46,1 & $110,0 \mathrm{~A}$ & $81,5 \mathrm{~B}$ & 95,7 & $62,3 \mathrm{~A}$ & $44,1 \mathrm{~B}$ & 53,2 & $172,2 \mathrm{~A}$ & $125,6 \mathrm{~B}$ & 148,9 & 57,7 & 57,2 & 57,5 \\
\hline \multirow{2}{*}{$\mathrm{Zn}$} & \multicolumn{3}{|c|}{ EFAZn $\left(10^{-5}\right)$} & \multicolumn{3}{|c|}{ EFTZn } & \multicolumn{3}{|c|}{ EFUZn } & & & & & & \\
\hline & Catuaí & Conilon & Média & Catuaí & Conilon & Média & Catuaí & Conilon & Média & & & & & & \\
\hline \multicolumn{16}{|l|}{$\mu \mathrm{mol} \mathrm{L} \mathrm{L}^{-1}$} \\
\hline 0,4 & 3,02 & 2,24 & $2,63 \mathrm{~b}$ & $0,825 a$ & $0,783 \mathrm{a}$ & 0,804 & 157634 & 182147 & $169890 a$ & & & & & & \\
\hline 1,3 & 6,00 & 2,70 & $4,35 a$ & $0,816 a$ & $0,686 \mathrm{~b}$ & 0,751 & 90483 & 147465 & $118974 b$ & & & & & & \\
\hline 2,3 & 6,73 & 4,00 & $5,35 \mathrm{a}$ & $0,655 \mathrm{~b}$ & $0,644 \mathrm{~b}$ & 0,650 & 78251 & 108659 & $93455 \mathrm{c}$ & & & & & & \\
\hline Média & $5,25 \mathrm{~A}$ & $2,98 \mathrm{~B}$ & 4,11 & $0,765 \mathrm{~A}$ & $0,704 \mathrm{~B}$ & 0,735 & $108890 \mathrm{~B}$ & $146090 \mathrm{~A}$ & 127440 & & & & & & \\
\hline
\end{tabular}

Médias seguidas pela mesma letra minúscula na coluna não diferem (Duncan $5 \%$ ); Médias seguidas pela mesma letra maiúscula na linha não diferem (ANOVA 5\%). 
Apenas os teores de $\mathrm{Zn}$ nas folhas inferiores $(\mathrm{ZnFl})$ e no caule $(\mathrm{ZnC})$ do Catuaí e nas raízes $(\mathrm{ZnR})$ dos dois cultivares foram influenciados pelo fornecimento de $Z n$ na solução nutritiva $(P<0,01)$. Assim, o teor médio de $\mathrm{Zn}$ nas folhas superiores foi de $6,8 \mathrm{mg} \mathrm{kg}^{-1}$, enquanto que o valor médio de $\mathrm{ZnFI}$ e $\mathrm{ZnC}$ do Conilon foi de $4,5 \mathrm{mg} \mathrm{kg}^{-1}$ e $10,0 \mathrm{mg} \mathrm{kg}^{-1}$ respectivamente. Os maiores valores de $\mathrm{ZnC}$ do Catuaí e $\mathrm{ZnR}$ dos dois cultivares foram observados na maior dose de $\mathrm{Zn}\left(2,3 \mu \mathrm{mol} \mathrm{L}^{-1}\right)$, enquanto que o máximo valor de $\mathrm{ZnFI}$ no Catuaí foi registrado na dose intermediária de $\mathrm{Zn}$ (Tabela 1).

Os conteúdos de $Z n$ no caule $(C Z n C)$, parte aérea $(\mathrm{CZnPA})$ e raízes $(\mathrm{CZnR})$ aumentaram com o fornecimento de zinco $(P<0,01)$. No Conilon, os maiores $\mathrm{CZnF}$ e CZnPA foram encontrados nas plantas cultivadas em soluções nutritivas mais concentradas em zinco, enquanto que os maiores $\mathrm{CZnF}$ e CZnPA no Catuaí foram obtidos com a dose intermediária de $Z n$. Os maiores CZnC e CZnR foram observados em plantas cultivadas sob a dose de $2,3 \mu \mathrm{mol} \mathrm{L}^{-1}$ nos dois cultivares.

O comportamento do conteúdo de $\mathrm{Zn}$ nas partes da planta entre o Catuaí e o Conilon seguiu o mesmo padrão em relação aos teores de $Z n$ em folhas e caule. O maior teor de $\mathrm{Zn}$ nas raízes do Catuaí e a maior produção de MSR do Conilon fizeram com que nenhuma diferença fosse registrada entre o CZnR dos dois cultivares

\section{Teores e conteúdo de Fósforo}

Catuaí apresentou maiores teores de fósforo nas folhas inferiores (PFI) $\left(1,6 \mathrm{~g} \mathrm{~kg}^{-1}\right)$ e na raiz $(P R)(1,3$ $\left.\mathrm{g} \mathrm{kg}^{-1}\right)$ que o Conilon $(P<0,01)$, que apresentou valores médios de PFI e PR iguais a $1,0 \mathrm{~g} \mathrm{~kg}^{-1}$ e $1,1 \mathrm{~g} \mathrm{~kg}^{-1}$, respectivamente (Tabela 2). $O$ conteúdo de $P$ em folhas inferiores (CPFI) e folhas (CPF) refletiu as diferenças na concentração e foram igualmente maiores no Catuaí $(P<0,05)$. O teores de fósforo nas folhas superiores (PFS) e no caule (PC) não diferiram entre os cultivares, e apresentaram valores médios de $1,3 \mathrm{~g} \mathrm{~kg}^{-1}$ e $1,2 \mathrm{~g}$ $\mathrm{kg}^{-1}$, respectivamente.

Os teores de PFS, PFI, PC e PR, bem como o conteúdo de fósforo nas folhas superiores (CPFS), CPFI, CPF, conteúdo de fósforo no caule (CPC), conteúdo de fósforo na raiz (CPR) e na parte aérea (CPPA) não foram influenciados pelas concentrações de $\mathrm{Zn}$ da solução nutritiva, demonstrando que nas concentrações empregadas não houve interação Zn-P para os cultivares de café.

\section{Eficiência de Absorção}

Catuaí apresentou maior eficiência de absorção de Zn (EFAZn) em condições de solução nutritiva que o Conilon $(P<0,01)$, absorvendo, em média e no período considerado, $52,5 \mu \mathrm{g}$ (ou $5,25.10^{-5} \mathrm{~g}$ ) do elemento por grama de matéria seca de raízes, enquanto que o Conilon, absorveu 29,8 $\mu \mathrm{g}$ por grama de matéria seca de raízes, no mesmo período (Tabela 1). O Conilon apresentou um sistema radicular maior, o que pode compensar em parte sua menor eficiência de absorção de $\mathrm{Zn}$.

Para ambos os cultivares, a EFAZn aumentou com o aumento das concentrações de $\mathrm{Zn}$ da solução nutritiva, resultando em aumento do conteúdo de $\mathrm{Zn}$ na planta. A produção de matéria seca pelo sistema radicular não foi influenciada pelas doses de $\mathrm{Zn}$, o que corrobora o aumento de conteúdo resultante de maior eficiência de absorção.

As diferentes concentrações de $\mathrm{Zn}$ da solução nutritiva não afetaram a eficiência de absorção de fósforo (Tabela 2), evidenciando que nas condições experimentais não ocorreu interação Zn-P. Houve diferença entre cultivares $(P<0,01)$, sendo o Catuaí mais eficiente na absorção de $P\left(7,50 \mathrm{mg} \mathrm{g}^{-1}\right.$ de matéria seca de raiz) que o Conilon (4,84 $\mathrm{mg} \mathrm{g}^{-1}$ de matéria seca de raiz).

\section{Eficiência de Translocação}

O Catuaí apresentou maior eficiência de translocação de zinco $(E F T Z n)$ que o Conilon $(P<0,01)$. O Catuaí translocou proporcionalmente mais $\mathrm{Zn}$ para a parte aérea que o Conilon, ou seja para cada mg de $\mathrm{Zn}$ absorvido, $0,765 \mu \mathrm{g}$ foram transportados para a parte aérea do Catuaí, enquanto que para o Conilon esse transporte foi de $0,704 \mu \mu_{\mu g^{-1}}$ (Tabela 1). Tanto no Catuaí como no Conilon, o aumento das concentrações de zinco da solução nutritiva reduziu a EFTZn $(P<0,01)$, o que resultou em maior concentração e acúmulo de $Z n$ nas raízes nas maiores concentrações de $\mathrm{Zn}$ (Tabela 1).

A eficiência de translocação de fósforo (EFTP) foi diferente entre os cultivares $(P<0,01)$. O Catuaí apresentou valor médio de EFTP maior que o Conilon (Tabela 2). O Catuaí se mostrou mais eficiente para a translocação de fósforo que o Conilon, sendo que para cada $10 \mathrm{mg}$ de $\mathrm{P}$ absorvidos, $8,20 \mathrm{mg}$ de $\mathrm{P}$ foram transportados para a parte aérea. Para o Conilon, esta proporção foi de 10:7,66.

As concentrações de $\mathrm{Zn}$ empregadas não afetaram a eficiência de translocação de $P$ dos cultivares em estudo.

\section{Eficiência de Utilização}

Embora menos eficiente na absorção e na translocação de $\mathrm{Zn}$ para a parte aérea, o Conilon apresentou maior eficiência de utilização de Zn (EFUZn) $(P<0,01)$, isto é, produziu em média $0,146 \mathrm{~g}$ de matéria seca por mg de $\mathrm{Zn}$ absorvido, enquanto que o Catuaí produziu $0,108 \mathrm{~g}$ de matéria seca para cada $\mu \mathrm{g}$ de $\mathrm{Zn}$ absorvido (Tabela 1). O aumento das concentrações de Zn para ambos os cultivares reduziu a EFUZn $(P<0,01)$.

$A$ eficiência de utilização de fósforo (EFUP) não foi afetada pelas concentrações de $\mathrm{Zn}$ em que as plantas foram cultivadas, porém os cultivares diferiram quanto à eficiência de utilização de $\mathrm{P}(P<0,01)$. O Conilon foi mais 
Tabela 2 - Altura de caule $(A C)$, área foliar $(A F)$, número de ramificações laterais (NRL), teores de fósforo nas folhas inferiores (PFI), folhas superiores (PFS), caule (PC) e raiz (PR), conteúdos de fósforo nas folhas inferiores $(C P F I)$, folhas superiores (CPFS), folhas (CPF), caule (CPC), parte aérea (CPPA) e raiz (CPR), e eficiências de absorção (EFAP), translocação (EFTP) e utilização (EFUP) de fósforo de plantas de café dos cultivares Catuaí e Conilon cultivados em solução nutritiva com diferentes concentrações de zinco.

\begin{tabular}{|c|c|c|c|c|c|c|c|c|c|c|c|c|c|c|c|}
\hline \multirow{2}{*}{$\mathrm{Zn}$} & \multicolumn{3}{|c|}{$A C$} & \multicolumn{3}{|c|}{$\mathrm{AF}$} & \multicolumn{3}{|c|}{$\mathrm{NF}$} & \multicolumn{3}{|c|}{ NRL } & & & \\
\hline & Catuaí & Conilon & Média & Catuaí & Conilon & Média & Catuaí & Conilon & Média & Catuaí & Conilon & Média & & & \\
\hline \multicolumn{4}{|c|}{ } & \multicolumn{9}{|c|}{ m } & & & \\
\hline 0,4 & 27,5 & 31,9 & 29,7 & 3477,6 & 2946,4 & 3212,0 & 54,3 & 37,0 & 45,7 & 2,50 & 0,67 & $1,58 \mathrm{~b}$ & & & \\
\hline 1,3 & 28,4 & 31,8 & 30,1 & 3562,3 & 3349,0 & 3455,7 & 60,3 & 33,0 & 46,7 & 3,17 & 0,67 & $1,92 a b$ & & & \\
\hline 2,3 & 28,4 & 34,7 & 31,6 & 3657,3 & 3818,8 & 3788,1 & 61,0 & 38,3 & 49,7 & 3,67 & 1,33 & $2,50 \mathrm{a}$ & & & \\
\hline Média & $28,1 \mathrm{~B}$ & $32,6 \mathrm{~A}$ & 30,4 & 3565,7 & 3371,4 & 3468,6 & $58,5 \mathrm{~A}$ & $36,1 \mathrm{~B}$ & 47,3 & $3,10 \mathrm{~A}$ & $0,90 \mathrm{~B}$ & 2,00 & & & \\
\hline \multirow{2}{*}{$\mathrm{Zn}$} & \multicolumn{3}{|c|}{$\mathrm{PFI}$} & \multicolumn{3}{|c|}{ PFS } & \multicolumn{3}{|c|}{ PC } & \multicolumn{3}{|c|}{ PR } & \multicolumn{3}{|c|}{ CPFI } \\
\hline & Catuaí & Conilon & Média & Catuaí & Conilon & Média & Catuaí & Conilon & Média & Catuaí & Conilon & Média & Catuaí & Conilon & Média \\
\hline$\mu \mathrm{mol} \mathrm{L}^{-1}$ & \multicolumn{12}{|c|}{ - } & \multicolumn{3}{|c|}{ 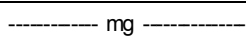 } \\
\hline 0,4 & 1,6 & 1,0 & 1,3 & 1,3 & 1,2 & 1,3 & 1,2 & 1,1 & 1,1 & 1,4 & 1,0 & 1,2 & 13,3 & 7,5 & 10,4 \\
\hline 1,3 & 1,7 & 1,0 & 1,4 & 1,3 & 1,2 & 1,3 & 1,2 & 1,1 & 1,1 & 1,3 & 1,2 & 1,2 & 14,8 & 8,9 & 11,8 \\
\hline 2,3 & 1,6 & 1,1 & 1,4 & 1,4 & 1,3 & 1,4 & 1,2 & 1,2 & 1,2 & 1,4 & 1,2 & 1,3 & 13,1 & 11,7 & 12,4 \\
\hline Média & $1,6 \mathrm{~A}$ & $1,0 \mathrm{~B}$ & 1,3 & 1,3 & 1,2 & 1,3 & 1,2 & 1,1 & 1,2 & $1,3 \mathrm{~A}$ & $1,1 \mathrm{~B}$ & 1,2 & $13,7 \mathrm{~A}$ & $9,4 \mathrm{~B}$ & 11,5 \\
\hline \multirow{2}{*}{$\mathrm{Zn}$} & \multicolumn{3}{|c|}{ CPFS } & \multicolumn{3}{|c|}{ CPF } & \multicolumn{3}{|c|}{ CPC } & \multicolumn{3}{|c|}{ CPPA } & \multicolumn{3}{|c|}{ CPR } \\
\hline & Catuaí & Conilon & Média & Catuaí & Conilon & Média & Catuaí & Conilon & Média & Catuaí & Conilon & Média & Catuaí & Conilon & Média \\
\hline$\mu \mathrm{mol} \mathrm{L}^{-1}$ & \multicolumn{15}{|c|}{ - } \\
\hline 0,4 & 7,9 & 7,9 & 7,9 & 21,2 & 15,4 & 18,3 & 4,1 & 3,1 & 3,6 & 25,3 & 18,6 & 21,9 & 6,2 & 5,2 & 5,7 \\
\hline 1,3 & 8,4 & 8,2 & 8,3 & 23,2 & 17,1 & 20,1 & 4,8 & 4,8 & 4,8 & 28,0 & 21,8 & 24,9 & 5,4 & 6,8 & 6,1 \\
\hline 2,3 & 10,2 & 9,7 & 9,9 & 23,2 & 21,3 & 22,3 & 4,6 & 7,2 & 5,9 & 27,8 & 28,5 & 28,6 & 6,1 & 9,0 & 7,5 \\
\hline Média & 8,8 & 8,6 & 8,7 & $22,5 \mathrm{~A}$ & $17,9 \mathrm{~B}$ & 20,2 & 4,5 & 4,8 & 4,8 & 27,0 & 23,0 & 25,0 & 5,9 & 7,0 & 6,4 \\
\hline \multirow{2}{*}{$\mathrm{Zn}$} & \multicolumn{3}{|c|}{$\operatorname{EFAP}\left(10^{-3}\right)$} & \multicolumn{3}{|c|}{ EFTP } & \multicolumn{3}{|c|}{ EFUP } & & & & & & \\
\hline & Catuaí & Conilon & Média & Catuaí & Conilon & Média & Catuaí & Conilon & $\overline{\text { Média }}$ & & & & & & \\
\hline \multicolumn{16}{|l|}{$\overline{\mu \mathrm{mol} \mathrm{L}^{-1}}$} \\
\hline 0,4 & 6,94 & 4,59 & 5,76 & 0,803 & 0,781 & 0,792 & 673 & 876 & 774 & & & & & & \\
\hline 1,3 & 7,86 & 4,78 & 6,32 & 0,837 & 0,751 & 0,794 & 699 & 843 & 771 & & & & & & \\
\hline 2,3 & 7,70 & 5,14 & 6,42 & 0,821 & 0,767 & 0,794 & 682 & 822 & 752 & & & & & & \\
\hline Média & $7,50 \mathrm{~A}$ & $4,84 \mathrm{~B}$ & 6,17 & $0,820 \mathrm{~A}$ & $0,766 \mathrm{~B}$ & 0,793 & $648 \mathrm{~B}$ & $847 \mathrm{~A}$ & 766 & & & & & & \\
\hline
\end{tabular}

Médias seguidas pela mesma letra minúscula na coluna não diferem (Duncan 5\%); Médias seguidas pela mesma letra maiúscula na linha não diferem (ANOVA 5\%).

eficiente na utilização de $P$, pois apresentou valor médio de EFUP maior que o Catuaí (Tabela 2). O Conilon produziu, em média e no período considerado, $0,847 \mathrm{~g}$ de matéria seca com $1 \mathrm{mg}$ de $P$, enquanto que o Catuaí produziu apenas $0,648 \mathrm{~g}$ de matéria seca com a mesma quantidade de fósforo.

\section{Interação Zn x P}

Diversas causas estão envolvidas na caracterização da interação entre zinco e fósforo. Estas causas estão baseadas em interações que ocorrem no solo e na planta. Neste experimento, o fornecimento de $\mathrm{Zn}$ aumentou teores e conteúdos de $\mathrm{Zn}$ na planta, mas não influenciou teores de $P$ em folhas, caules e raízes dos cultivares avaliados. O mesmo efeito foi observado em relação às eficiências de absorção, translocação e utilização de nutriente. Enquanto EFAZn, EFTZn e EFUZn foram influenciadas pela concentração de $Z n$ na solução nutritiva, EFAP, EFTP e EFUP não foram influenciadas pelas doses de Zn. Há autores (Ghoneim e Brussler, citado por Marschner, 1986) que afirmam que a interação Zn-P não ocorre a nível de planta e sim no solo. $O$ presente trabalho não encontrou interação entre Zn e P em plantas cultivadas em solução nutritiva, e novos estudos são necessários com maiores doses de
Zn em solução nutritiva para averiguar se esta interação ocorre somente no solo ou na planta ou em ambas.

\section{AGRADECIMENTO}

Ao laboratorista Domingos Sávio pelo auxílio durante as análises químicas de tecidos vegetais realizadas neste trabalho.

\section{REFERÊNCIAS BIBLIOGRÁFICAS}

BINGHAM, F.T. Relations between phosphorus and micronutrients in plants. Soil Science Society of America Proceedings, v.27, p.389-391, 1963

GALLO, J.R.; HIROCE, R.; COELHO, F.A.S. Levantamento do estado nutricional de cafezais de São Paulo, pela análise foliar. I. Solo massapé - salmourão. Bragantia, v.26, p.103-117, 1967.

GALLO, J.R.; HIROCE, R.; BATAGLIA, O.C.; MORAES, F.R.P. Levantamento de cafezais do Estado de São Paulo pela análise química foliar: II. Solos Podzolizados de Lins e Marília, Latossolo Roxo e Podzólico Vermelho-Amarelo orto. Bragantia, v.29, p.237-247, 1970.

GARCIA, A.W.R.; CORREA, J.B.; FREIRE, A.C.F. Levantamento das características químicas dos solos e estado nutricional das lavouras cafeeiras do sul de Minas. In: CONGRESSO BRASILEIRO DE PESQUISAS CAFEEIRAS, 10., Poços de Caldas, 1983. Anais. Rio de Janeiro: IBC/GERCA, 1983. p.5-8.

INSTITUTO BRASILEIRO DO CAFÉ. Cultura do café no Brasil: manual de recomendações. 5.ed. Rio de Janeiro: IBC, 1985. 580p.

IGUE, K.; BORNEMISZA, E. El problema del Zn en suelos y plantas de regiones tropicales y de zonas templadas. Fitotecnia Latinoamericana, v.4, p.29-44, 1967. 
LOTT, W.L.; Mc CLUNG, A.C.; VITA, R.; GALLO, J.R. A survey of coffee fields in São Paulo and Paraná by foliar analysis. São Paulo: IBEC Research Institute, 1961. 72 p. (Boletim Técnico, 26).

MALAVOLTA, E.; FERNANDES, D.R.; ROMERO, J.P. Seja doutor do seu cafezal. Informações Agronômicas, v.64, p.1-12, 1993.

MARQUES, R. Efeitos do fósforo e do zinco na nutrição e crescimento de porta-enxerto de seringueira (Hevea brasiliensis Muell Arg.). Lavras, 1990. 90p. Tese (Mestrado) - Universidade Federal de Lavras.

MARSCHNER, H. Mineral nutrition of higher plants. London: Academic Press, 1986. 674p.

OLSEN, S.R. Micronutrient interactions. In: MORTVEDT, J.J.; GIORDANO, P.M.; LINDSAY, W.L. (Ed.) Micronutrients in agriculture. Madison: SSSA, 1972. p.243-264.
PASRICHA, N.S.; BADDESHA, H.S.; AULAKH, M.S.; NAYYAR, V.K. The zinc quantity: intensity relationships in four different soils as influenced by phosphorus. Soil Science, v.143, p.1-4, 1987.

SOUZA, E.C.A.; FERREIRA, M.E. Micronutrientes no solo: zinco. In: FERREIRA, M.E.; CRUZ, C.P. Micronutrientes na agricultura. Piracicaba: Potafós; CNPq, 1991. p.219-242.

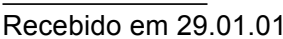

